\title{
CRESCIMENTO JUVENIL DE Pinus taeda EM FUNÇÃO DO ESPAÇO VITAL DE CRESCIMENTO
}

\author{
Mário Takao Inoue ${ }^{1}$, Afonso Figueiredo Filho ${ }^{1}$, Antonio José de Araújo ${ }^{1}$, Rodrigo Lima ${ }^{2}$ \\ ${ }^{1}$ Eng. Florestal, Dr., Depto. de Engenharia Florestal, UNICENTRO, Irati, PR, Brasil - takao@irati.unicentro.br; afig@ufpr.br \\ ${ }^{2}$ Eng. Florestal, Mestrando em Ciências Florestais, UNICENTRO, Irati, PR, Brasil - rodrigo_eng3@hotmail.com
}

Recebido para publicação: 06/07/2009 - Aceito para publicação: 21/06/2010

\begin{abstract}
Resumo
Avaliou-se o desempenho de Pinus taeda L. aos quatro anos de idade, plantado em diferentes espaçamentos, simulando espaços vitais entre $1 \mathrm{~m}^{2}$ e $16 \mathrm{~m}^{2}$. O experimento foi implantado em 2002, com mudas de Pinus taeda L. produzidas com sementes de pomar clonal. O delineamento experimental foi de blocos ao acaso, com cinco repetições e nove tratamentos (espaçamentos $1,0 \times 1,0 ; 2,0 \times 1,0 ; 2,0 \times 2,0 ; 3,0$ x 2,5; 3,0 x 3,0;3,0 x 3,5; 4,0 x 3,0;4,0 x 3,5; 4,0 x 4,0 m), constituídos por 25 mudas e uma linha de bordadura. Não foram significantes as correlações entre espaço vital e altura, nem entre espaço vital e DAP. O diâmetro dos ramos, entretanto, está correlacionado com o espaço vital. Existe correlação entre altura e DAP e entre DAP e diâmetro de ramos. A altura das árvores variou entre 4,08 m e 4,60 m, e o DAP entre $5,65 \mathrm{~cm}$ e 7,69 cm. Não se detectou diferenças significantes no crescimento da altura e do DAP. O espaço vital afetou o crescimento dos ramos, cujos diâmetros variaram entre 11,45 mm e 19,01 mm. Quanto maior o espaço vital, maiores os diâmetros dos ramos.

Palavras-chave: Espaçamento; diâmetro de ramos; desrama.
\end{abstract}

\begin{abstract}
Growth of Pinus taeda at early age as function of the growing space. It was evaluated the growth of four years old Pinus taeda L. planted at nine different spacings $(1.0 \times 1.0 ; 2.0 \times 1.0 ; 2.0 \times 2.0 ; 3.0 \times$ $2.5 ; 3.0 \times 3.0 ; 3.0 \times 3.5 ; 4.0 \times 3.0 ; 4.0 \times 3.5 ; 4.0 \times 4.0 \mathrm{~m}$ ), simulating vital spaces ranging from $1 \mathrm{~m}^{2}$ to $16 \mathrm{~m}^{2}$. The trial was established in 2002 with seedlings of Pinus taeda L. originated from a clonal seed orchard. It was used randomized blocks with five replications, each plot with 25 seedlings, excluding a border line. There were no significant correlation between growing space and height or between growing space and $\mathrm{DBH}$. The diameter of branches was correlated with growing space. Positive correlations were obtained between height and $\mathrm{DBH}$ and between branch diameter and $\mathrm{DBH}$. Tree height ranged from $4.08 \mathrm{~m}$ to $4.60 \mathrm{~m}$ and the DBH from $5.65 \mathrm{~cm}$ e $7.69 \mathrm{~cm}$ and there were no significant differences for both variables. The growing space affected branch growth. Larger growing spaces resulted in larger branch diameter. Branch diameters ranged from $11.45 \mathrm{~mm}$ to $19.01 \mathrm{~mm}$. Keywords: Spacing; diameter of branches; pruning.
\end{abstract}

\section{INTRODUÇÃO}

O setor florestal brasileiro, principalmente os segmentos de celulose e madeira serrada, depende fundamentalmente de reflorestamentos com espécies introduzidas, quase que exclusivamente dos gêneros Pinus e Eucalyptus. Em plantios na região Sul, considerando as condições climáticas mais amenas, predominam Pinus taeda L. e Pinus elliottii Engelm.

No anuário divulgado pela Associação Brasileira de Produtores de Florestas Plantadas (ABRAF, 2008), consta que 95,3\% da produção sustentável de madeira em tora de Pinus encontram-se nas regiões Sul e Sudeste do Brasil. Mesmo com extensas áreas de plantio nessas regiões, a falta de madeira de qualidade no mercado preocupa empresários do ramo florestal, levando os produtores a procurar técnicas adequadas de manejo florestal para agregar maior valor ao produto final.

A definição de técnicas de manejo a empregar em plantios de Pinus spp. em relação ao objetivo da produção é um dos principais problemas da administração florestal em empresas de médio e grande porte (GOMES et al., 2002). 
Berger (2000) afirma que, em florestas de ciclo curto, o espaçamento pode afetar direta e rapidamente a qualidade da madeira produzida. Desde a era dos incentivos fiscais da década de 60 do século passado, o cultivo dessas espécies tem sido feito com espaçamentos que variam entre $2,0 \mathrm{~m} \times 2,0 \mathrm{~m}$ até $3,0 \mathrm{~m} \mathrm{x} \mathrm{3,0} \mathrm{m,} \mathrm{correspondendo} \mathrm{a} \mathrm{um} \mathrm{espaço} \mathrm{vital} \mathrm{entre} 4 \mathrm{~m}^{2}$ e $9 \mathrm{~m}^{2}$ para cada árvore.

Segundo Couto et al. (2002), existe um consenso em relação ao efeito do espaçamento sobre as variáveis de crescimento da árvore e do povoamento. Dessa forma, os menores espaçamentos resultam em menor diâmetro quadrático, maior área basal por hectare, maior volume total por hectare e menor porcentagem de sobrevivência.

$\mathrm{Na}$ literatura específica, encontramos vários estudos relacionados ao espaçamento para produção de Pinus taeda. Entre eles, podem-se citar Harms e Langdon (1976), Pienaar e Shiver (1993), Gomes et al. (1997), Sanquetta et al. (1998), Baldwin Jr. et al.( 2000), Harms et al. (2000), Will et al. (2001), Radtke et al. (2003), Chies (2005) e Leite et al. (2006).

A qualidade do sítio florestal é fator importante na tomada de decisões quanto ao regime de manejo a ser adotado. Dependendo das condições do sítio e do espaçamento adotado, o primeiro desbaste para Pinus taeda L. deve ocorrer entre 6 e 8 anos. Determinar o espaçamento ideal para o plantio de Pinus é um fator fundamental, pois irá influenciar diretamente na qualidade e destinação final da madeira. Essa decisão afeta a formação das florestas, os tratamentos culturais, a qualidade da madeira, a colheita florestal e, consequentemente, os custos da produção (LEITE et al., 2006).

Atualmente, com o mercado exigindo maior demanda em madeira livre de nós, fator que amplia seu aproveitamento para fins mais nobres e bem remunerados, é comum a operação de desrama, quase sempre executada sem maiores critérios técnicos. No entanto, devido à escassez de madeira desramada no mercado, as indústrias aproveitam o mesmo tipo de tora que as demais no seu processo industrial, para produção de painéis colados, "clear blocks", "finger joint" etc., retirando os nós na sua manufatura e conseguindo, com isso, alto valor para tais produtos (SCOLFORO et al., 1997).

As relações entre o espaço vital disponível e o crescimento do Pinus spp. ainda não são bem conhecidas. Sobretudo, não se conhecem possíveis interações que possam existir entre o crescimento diametral e em altura com o crescimento dos ramos verticilares. Dessa forma, estabeleceram-se as seguintes hipóteses de trabalho, considerando a idade das árvores:

a) Diferentes espaços vitais afetam o crescimento em altura, DAP e diâmetro dos ramos.

b) As variáveis altura, DAP e diâmetro dos ramos estão correlacionadas entre si.

\section{MATERIAL E MÉTODOS}

\section{Área de estudo}

O município de Irati encontra-se a 156 km de Curitiba, capital do Paraná. Está localizado na região centro-sul do estado, entre as coordenadas geográficas $25^{\circ} 27^{\prime} 56^{\prime \prime}$ de latitude sul, com interseção com o meridiano 50 37'51'" de longitude oeste. Encontra-se na região dos Campos Gerais, no segundo planalto, em meio à Floresta Ombrófila Mista.

O clima da região, conforme a classificação de Köppen, é definido como $C f b$ Subtropical Úmido Mesotérmico, de verões frescos, geadas severas e frequentes, sem estação seca. A média das temperaturas dos meses mais frios é inferior a $11{ }^{\circ} \mathrm{C}$, e dos meses mais quentes, inferior a $24,2{ }^{\circ} \mathrm{C}$.

As precipitações ocorrem durante todos os meses do ano. A precipitação média mensal no município é de 193,97 mm. Na região predominam solos sílticos argilosos, litólicos, coluviais e saprolíticos (IRATI, 2008).

\section{Características do experimento}

Implantou-se em 2002, no campus universitário de Irati, um experimento com mudas produzidas com sementes de um pomar clonal de Pinus taeda L., cedidas pela empresa Klabin do Paraná. Como tratamentos, foram considerados nove espaçamentos, simulando espaços vitais de $1 \mathrm{~m}^{2}$ a $16 \mathrm{~m}^{2}$ (Tabela 1). O ensaio foi instalado em blocos ao acaso (Figura 1), com cinco repetições e parcelas experimentais constituídas por 25 mudas, considerando uma linha de bordadura em cada parcela.

O preparo de solo foi feito por aração e o plantio foi manual, com perfuração de covas com o uso de sacho. $\mathrm{O}$ controle de formigas cortadeiras foi feito por ocasião do plantio, com o uso de iscas à base de $10 \mathrm{~g}$ por $100 \mathrm{~m}^{2}$ de terreno. 
A limpeza da área experimental foi feita um ano após o plantio, por meio de coroamento ao redor das plantas e capina nos espaços entre blocos, sendo repetida anualmente.

Em 2006, aos quatro anos de idade, foi realizada a primeira desrama das árvores, visando a obtenção de um núcleo nodoso de aproximadamente $5 \mathrm{~cm}$. A operação foi aplicada em todas as árvores até o verticilo imediatamente abaixo de $1,3 \mathrm{~m}$ do solo.

Tabela 1. Caracterização dos tratamentos.

Table 1. Characterization of treatments.

\begin{tabular}{lccc}
\hline Tratamento & Espaçamento $(\mathbf{m})$ & Área útil por árvore $\left(\mathbf{m}^{2}\right)$ & Número de árvores medidas \\
\hline 1 & $1,0 \times 1,0$ & 1,0 & 25 \\
2 & $2,0 \times 1,0$ & 2,0 & 25 \\
3 & $2,0 \times 2,0$ & 4,0 & 25 \\
4 & $3,0 \times 2,5$ & 7,5 & 25 \\
5 & $3,0 \times 3,0$ & 9,0 & 25 \\
6 & $3,0 \times 3,5$ & 10,5 & 25 \\
7 & $4,0 \times 3,0$ & 12,0 & 25 \\
8 & $4,0 \times 3,5$ & 14,0 & 25 \\
9 & $4,0 \times 4,0$ & 16,0 & 25 \\
\hline
\end{tabular}

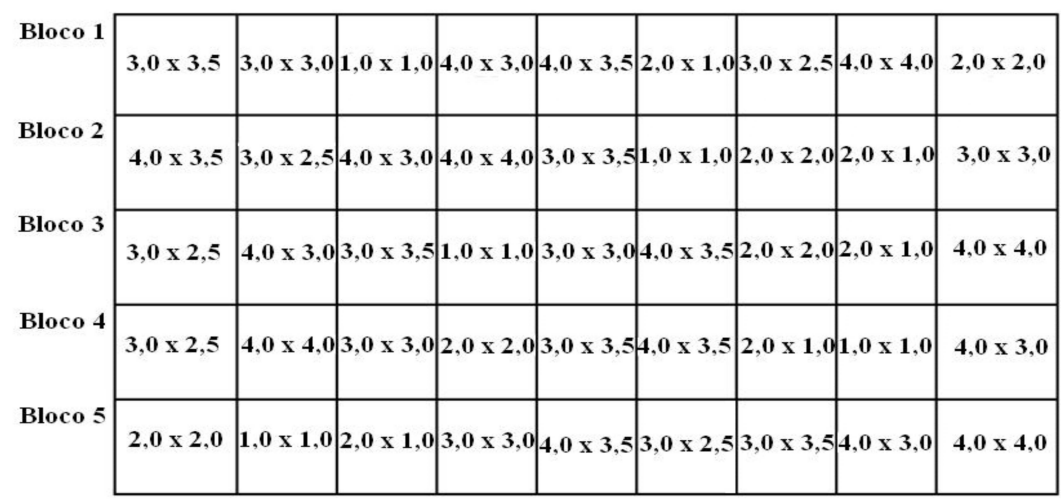

Figura 1. Layout do experimento.

Figure 1. Experimental layout.

\section{Obtenção dos dados}

A cada ano, até 2005, foram medidas apenas as alturas das mudas. Em 2006 foram medidas a altura total, DAP (diâmetro a 1,3 m do solo) e diâmetro dos ramos do verticilo mais próximo ao DAP, por ocasião da desrama. Para efeito de avaliação por árvore, calculou-se a média dos diâmetros de todos os ramos pertencentes ao verticilo e das demais variáveis medidas.

\section{Análise estatística}

Foram calculadas as correlações entre as variáveis altura, DAP e diâmetro de ramos e destas com o espaço vital, utilizando-se o coeficiente de correlação linear de Pearson, calculado para um nível de significância $\alpha=0,01$. Os valores das variáveis medidas foram submetidos ao teste de Bartlett para verificação da homogeneidade de variâncias, análise de variância (ANOVA) e teste de Tukey para comparação entre médias para um nível de $5 \%$ de probabilidade.

\section{RESULTADOS E DISCUSSÃO}

\section{Correlação linear de Pearson}

$\mathrm{Na}$ tabela 2 constam os valores referentes às correlações simples entre variáveis (altura, DAP e diâmetro do ramo) e o espaço vital. Não foram significativas as correlações entre espaço vital e altura das 
árvores, nem entre espaço vital e DAP. O diâmetro dos ramos, entretanto, foi correlacionado com o espaço vital, ou seja, quanto maior o espaço vital, maiores os diâmetros dos ramos verticilares. O baixo valor encontrado para a correlação $(\mathrm{r}=0,3052$ probabilidade $p<0,01)$, embora significante, não permite concluir sobre a real existência de tal correlação. No entanto pode indicar uma tendência verdadeira, pois é fato conhecido que árvores menos sombreadas desenvolvem ramos mais grossos (WEISKITTEL et al, 2007), condição caracterizada pelos espaçamentos menos apertados do presente estudo.

Tabela 2. Coeficiente de correlação linear de Pearson das variáveis em relação ao espaço vital, entre as variáveis e das variáveis em relação ao diâmetro do ramo.

Table 2. Linear correlation coefficient of Pearson for variables in relation of vital space, between the variables and the variables in relation to branch diameter.

\begin{tabular}{lcc}
\hline Variáveis & $\mathbf{R}$ & $\boldsymbol{p}$ \\
\hline Altura x Espaço vital & 0,0083 & 0,5496 \\
DAP x Espaço vital & 0,0716 & 0,0666 \\
Diâmetro do ramo x Espaço vital & 0,3052 & $0,0000^{* *}$ \\
Altura x DAP & 0,7831 & $0,0000^{* *}$ \\
Altura x Diâmetro do ramo & 0,2413 & $0,0006^{* *}$ \\
DAP x Diâmetro do ramo & 0,5564 & $0,0000^{* *}$ \\
\hline
\end{tabular}

**Correlação significativa a $p<0,01$.

A mais alta correlação encontrada foi entre altura e DAP $(\mathrm{r}=0,7831$ probabilidade $p<0,01)$. Essa é uma correlação conhecida e esperada, com indivíduos mais altos apresentando diâmetros maiores, como encontrado por Barrichelo et al. (1977), com valor significante de $\mathrm{r}=0,86$ para Pinus taeda $\mathrm{L}$. aos quatro anos de idade. Evidenciou-se existir correlação positiva entre DAP e diâmetro de ramos $(\mathrm{r}=0,5564$ probabilidade $p<0,01)$. Parte dessa correlação pode ser influenciada pela correlação significante entre espaço vital e diâmetro de ramos, já que não foi detectado existir correlação entre DAP e espaço vital. Embora tenha sido encontrada correlação significante $(\mathrm{r}=0,2413$ probabilidade $p<0,01)$ entre altura e diâmetro de ramos, o baixo valor do coeficiente revela não existir uma real correlação entre tais variáveis.

\section{Altura, DAP e diâmetro de ramos}

O teste de Bartlett para homogeneidade das variâncias permitiu conduzir a análise de variância (ANOVA). A tabela 3 mostra os resultados obtidos no teste. Como o nível de significância para todas as variáveis ultrapassou o valor crítico de alfa $(0,05)$, fica evidenciada que as variâncias são homogêneas e que, portanto, podem ser submetidas à análise de variância.

Tabela 3. Valores do qui-quadrado $\left(\chi^{2}\right)$ obtidos no teste de Bartlett e seus respectivos níveis de significância para cada variável.

Table 3. Values of chi-square $(\chi 2)$ obtained by Bartlett-test with correspondent signficance level.

\begin{tabular}{lcc}
\hline Variáveis & Valor $(\chi 2)$ & Nível de significância $(\boldsymbol{\alpha})$ \\
\hline Altura $(\mathrm{m})$ & 3,5701 & 15,5073 \\
DAP $(\mathrm{cm})$ & 2,3463 & 15,5073 \\
Diâmetro de ramo $(\mathrm{mm})$ & 5,6651 & 15,5073 \\
\hline
\end{tabular}

Na tabela 4 estão apresentados os resultados das análises de variâncias calculadas para altura, diâmetro à altura do peito (DAP) e diâmetro dos ramos. As médias de altura das árvores variaram de $4,08 \mathrm{~m}$ a 4,60 m, não se detectando diferença significante no crescimento em altura para os espaçamentos testados. Os valores médios de DAP estiveram entre $5,65 \mathrm{~cm}$ e 7,69 $\mathrm{cm}$, não se detectando diferença significante entre os tratamentos. Foi detectada diferença altamente significante para o diâmetro médio dos ramos do primeiro verticilo remanescente após a desrama. Os valores médios dessa variável ficaram entre $11,45 \mathrm{~mm}$ e $19,01 \mathrm{~mm}$, e a diferença estatística foi comprovada apenas entre os tratamentos 1 e 8 , respectivamente, espaçamento de $1,0 \mathrm{~m}$ x $1,0 \mathrm{~m} \mathrm{e} \mathrm{4,0} \mathrm{m} \mathrm{x} \mathrm{3,5} \mathrm{m.} \mathrm{Os} \mathrm{tratamentos} \mathrm{4,} 7$ e 8 apresentaram os menores valores de C.V. para as variáveis altura, DAP e diâmetro dos ramos. Em relação apenas ao diâmetro dos ramos, o tratamento 5 apresentou o mais baixo coeficiente de variação, conforme tabela 4 . 
Tabela 4. Valores médios de altura, DAP e diâmetro de ramos de Pinus taeda L. aos quatro anos de idade. Table 4. Average values of height, DBH and branch diameter growth for Pinus taeda L. at age four.

\begin{tabular}{lcccccc}
\hline Tratamento & Altura $(\mathbf{m})$ & $\mathbf{C . V}$ & DAP $(\mathbf{c m})$ & $\mathbf{C . V}$ & Diâmetro de ramo $(\mathbf{m m})$ & C.V. \\
\hline 1 & $4,38 \mathrm{a}$ & 0,19 & $5,65 \mathrm{a}$ & 0,22 & $11,45 \mathrm{~b}$ & 0,20 \\
2 & $4,45 \mathrm{a}$ & 0,16 & $6,22 \mathrm{a}$ & 0,19 & $13,61 \mathrm{ab}$ & 0,31 \\
3 & $4,48 \mathrm{a}$ & 0,14 & $6,82 \mathrm{a}$ & 0,20 & $14,68 \mathrm{ab}$ & 0,24 \\
4 & $4,60 \mathrm{a}$ & 0,10 & $7,43 \mathrm{a}$ & 0,12 & $17,87 \mathrm{a}$ & 0,07 \\
5 & $4,39 \mathrm{a}$ & 0,15 & $6,95 \mathrm{a}$ & 0,22 & $17,22 \mathrm{ab}$ & 0,11 \\
6 & $4,31 \mathrm{a}$ & 0,12 & $6,80 \mathrm{a}$ & 0,19 & $18,39 \mathrm{a}$ & 0,16 \\
7 & $4,40 \mathrm{a}$ & 0,09 & $7,33 \mathrm{a}$ & 0,14 & $17,75 \mathrm{a}$ & 0,15 \\
8 & $4,57 \mathrm{a}$ & 0,10 & $7,69 \mathrm{a}$ & 0,13 & $19,01 \mathrm{a}$ & 0,14 \\
9 & $4,08 \mathrm{a}$ & 0,19 & $6,51 \mathrm{a}$ & 0,27 & $16,83 \mathrm{ab}$ & 0,19 \\
\hline \\
\multicolumn{2}{l}{ Valores nas colunas seguidos pela mesma letra não diferem estatisticamente entre si ao nível de $p<0,05$. C.V.: coeficiente de } \\
variação.
\end{tabular}

Avaliando um plantio de Pinus taeda aos 12 anos de idade no município de Jaguariaíva, região norte do Paraná, Sanquetta et al. (2003) constataram maiores diâmetros (DAP) quanto maior o espaço vital (naquele caso, até $11 \mathrm{~m}^{2}$ ), sendo a altura não afetada pelo espaçamento, fato já observado em outros estudos realizados na mesma região (GOMES et al., 1997; SANQUETTA et al., 1998). Também Leite et al. (2006), estudando o efeito do espaçamento e da idade sobre as variáveis de povoamentos de Pinus taeda L. no planalto serrano de Santa Catarina, não constataram efeito do espaço vital (entre $1,5 \mathrm{~m}^{2} \mathrm{e}$ $7,5 \mathrm{~m}^{2}$ ) sobre a altura das árvores até os oito anos de idade, verificando diferença de até $1,1 \mathrm{~m}$ entre tratamentos quando medidos aos 14 anos. Esses autores confirmaram que espaçamentos mais amplos proporcionam diâmetros maiores.

Até os quatro anos de idade, o crescimento das árvores do presente estudo apresentou um incremento médio de mais de um metro de altura por ano e mais de um centímetro de DAP por ano. A tendência dos resultados presentes aponta para uma confirmação dos dados encontrados na literatura. Nos estudos de Leite et al. (2006) foi constatado que somente a partir do décimo ano iniciou-se o efeito do espaço vital sobre a altura das árvores.

Os espaços vitais utilizados neste estudo, que variaram entre $1 \mathrm{~m}^{2}$ até $16 \mathrm{~m}^{2}$ para cada árvore, os quais não promoveram efeitos significantes no crescimento em altura e no DAP até a idade de quatro anos, indicam claramente que o ponto de inflexão nos incrementos daquelas variáveis devido à concorrência por espaço vital ainda não foi atingido.

\section{CONCLUSÕES} se concluir:

Para as condições do estudo e até a idade de quatro anos após plantio, para Pinus taeda L., pode-

- Não existe correlação entre espaço vital e altura e entre espaço vital e DAP.

- Existe indício de correlação positiva entre espaço vital e diâmetro dos ramos.

- Árvores mais altas estão correlacionadas com DAP maiores.

- Árvores mais grossas apresentam ramos mais grossos.

- O espaçamento não afetou o crescimento do DAP e da altura total.

\section{REFERÊNCIAS}

ABRAF - ANUÁRIO ESTATÍSTICO DA ABRAF: ano base 2007/ABRAF. Brasília, 2008. p. 90.

BALDWIN JR., V. C.; PETERSON, K. D.; CLARKLLL, A.; FERGUSON, R. B.; STRUB, M. R.; BOWER, D. R. The effects of spacing and thinning on stand and tree characteristics of 38-year-old Loblolly Pine. Forest Ecology and Management, v. 137, p. 91-102, 2000.

BARRICHELO, L. E. G.; KAGEYAMA, P. Y.; SPELTZ, R. M.; BONISH, H. J.; BRITO, J. O.; FERREIRA, M. Estudos de procedências de Pinus taeda visando seu aproveitamento industrial. IPEF, n. 15 , p. $1-14,1977$. 
BERGER, R. Crescimento e qualidade da madeira de um clone de Eucalyptus saligna Smitch sob o efeito do espaçamento e da fertilidade. Santa Maria: UFSM, 2000. 106 p. Dissertação (Mestrado em Ciências Florestais) - Universidade Federal de Santa Maria.

CHIES, D. Influência do espaçamento sobre a qualidade e o rendimento da madeira serrada de Pinus taeda L. Curitiba: 2005. 123 p. Dissertação (Mestrado em Ciências Florestais) - Setor de Ciências Agrárias, Universidade Federal do Paraná.

COUTO, L.; MULLER, M. D.; DIAS, A. N.; TSUKAMOTO FILHO, A. A.; FONSECA, E. M. B.; CORRÊA, M. R. Espaçamento de plantio de espécie de rápido crescimento para dendroenergia. Belo Horizonte: CEMIG, 2002. 66 p.

GOMES, F. S.; SANQUETTA, C. R.; SCOLFORO, J. R.; GRAÇA, L. R.; MAESTRI, R. Efeitos do sítio e de cenários de custos e preços na análise de regimes de manejo com e sem desbastes em Pinus taeda $\mathrm{L}$. Cerne, v. 8, n. 1, p: 13-31, 2002.

GOMES, F. S.; MAESTRI, R.; SANQUETTA, C. R. Avaliação da produção em volume toral e sortimentos de povoamentos de Pinus taeda L. submetidos a diferentes condições de espaçamento inicial e sítio. Ciência Florestal, Santa Maria, v. 7, n. 1, p. 101-126, 1997.

HARMS, W. R.; LANGDON, O. G. Development of loblolly pine in dense stands. Forest Science, v. 22, p. 331-337, 1976.

HARMS, W. R.; WHITESELL, C. D.; DEBELL D. S. Growth and development of loblolly pine in a spacing trial planted in Hawaii. Forest Ecology and Management, v. 126, p. 13-14, 2000.

IRATI, Prefeitura Municipal. Clima. Disponível em: 〈http://www.irati.pr.gov.br/municipio/clima.asp>. Acessado em jul. 2008.

IRATI, Prefeitura Municipal. Tipos predominantes de Solos. Disponível em: <http://www.irati.pr.gov.br/municipio/geografia.asp>. Acessado em jul. 2008.

LEITE, H. G.; NOGUEIRA, G. S.; MOREIRA, A. M. Efeito do espaçamento e da idade sobre variáveis de povoamentos de Pinus taeda L. Árvore, Viçosa, n. 30, v. 4, p: 603-612, 2006.

PIENAAR, L. V.; SHIVER, B. D. Early results from an old-field loblolly pine spacing study in the Georgia piedmont with competition control. South. J. Appl. For., v. 17, p. 193-196, 1993.

RADTKE, P. J.; WESTFAll, J. A.; BURKHART, A. W. Conditioning a distance-dependent competition index to indicate the onset of intertree competition. Forest Ecology and Management, v. 175, p. 17-30, 2003.

SANQUETTA, C. R.; ARCE, J. E.; MELlO, A. A.; SILVA, E. Q.; BARTH FILHO, N.; MATOSKI, S. L. S. Produção de madeira livre de nós em povoamentos de Pinus taeda em função da densidade de plantio. Cerne, Lavras, v. 9, n. 2, p. 129-140, 2003.

SANQUETTA, C. R.; REZENDE, A. V.; GAIAD, D.; SCHAAF, L. B.; ZAMPIER, A. C. Produção de madeira para laminação em povoamentos de Pinus taeda submetidos a diferentes densidades e regimes de desbaste: uma abordagem experimental. Floresta, Curitiba, v. 28, n. 1/2, p. 80-96, 1998.

SCOLFORO, J. R. S.; MAESTRI, R. O manejo de florestas plantadas. In: SCOLFORO, J. R. S. Manejo florestal. Lavras: UFLA/FAEPE, 1997. Cap. 8. p. 313-379.

WEISKITTEL, A. R.; MAGUIRE, D. A.; MONSERUD, R. A. Response of branch growth and mortality to silvicultural treatments in coastal Douglas-fir plantations: Implications for predicting tree growth. Forest Ecology and Management, v. 251, n. 3, p. 182-190, 2007.

WILL, R. E.; BARRON, G. A.; BURKES, E. C.; SHIVER, B.; TESKEY, R. O. Relationship between intercepted radiation, net photosynthesis, respiration, and rate of stem volume growth of Pinus taeda and Pinus elliottii stands of different densities. Forest Ecology and Management, v. 154, p. 155-163, 2001. 